\title{
661 NEOANTIGEN-SPECIFIC CD4+ T CELLS IN HUMAN MELANOMA HAVE DIVERSE DIFFERENTIATION STATES AND CORRELATE WITH CD8+ T CELL, MACROPHAGE, AND B CELL FUNCTION
}

${ }^{1}$ Naina Singhi, 'Carolyn Shasha, 'Sylvia Lee, 'Julia Szeto, ${ }^{1}$ Ata Moshiri, ${ }^{1}$ Teresa Kim, ${ }^{2} J o h n$ Thompson, 'Scott Tykodi, 'Venu Pillarisetty, 'David Byrd, 'Kimberly Smythe, ${ }^{1}$ Shailender Bhatia, 'Evan Hall, 'Evan Newell, ${ }^{1}$ Raphael Gottardo, 'Stanley Riddell, ${ }^{1}$ Joshua Veatch*. ${ }^{1}$ Fred Hutchinson Cancer Research Center, Seattle, WA, United States; ${ }^{2}$ University of Washington, Seattle, WA, United States

Background Tumor-antigen specific CD4 $+\mathrm{T}$ cells are crucial for the efficacy of antibodies that block immune checkpoint proteins in mouse tumor models, but their activities in human tumor immunity are less clear. CD8 $+\mathrm{T}$ cells infiltrating human tumors, including those specific for tumor antigens, have been studied using single cell profiling techniques and exist in a variety of dysfunctional states. The transcriptional states of tumor-specific CD4 $+\mathrm{T}$ cells present in tumors and their potential contributions to the tumor microenvironment are less well understood.

Methods We used targeted single cell RNA sequencing and matching of $\mathrm{T}$ cell receptor (TCR) sequences to identify phenotypic signatures that discriminated tumor antigen- and viral antigen-specific $\mathrm{CD} 4+\mathrm{T}$ cells infiltrating human melanoma tumors in four patients. The presence of CD4+ T cells with these signatures was correlated with the number and phenotype of other immune cells in the tumor microenvironment in an extended cohort of 20 patients.

Results We identified 259 CD4+ T cells representing 40 different TCR clonotypes specific for 13 neoantigens and 108 cells representing 14 TCR clonotypes specific for self-antigens in four melanoma patients. High expression of CXCL13 defined conventional CD4 $+\mathrm{T}$ cells that recognize tumor associated neoantigens and self-antigens from bystander and viral antigen-specific CD4+ T cells. Tumor-reactive CD4 $+\mathrm{T}$ cells could be subdivided into clusters expressing memory and $\mathrm{T}$ follicular helper markers, and those expressing cytolytic markers and IFN-g. In an extended cohort of 20 patients with melanoma, the frequency of CXCL13 + CD4+ T cells in the tumor microenvironment correlated with the presence and proliferation of CD8 $+\mathrm{T}$ cells, the presence and maturation of $\mathrm{B}$ cells, the activation of interferon responsive genes in tumor associated macrophages, and patient survival. CD4+ T cells with similar transcriptional signatures were identified in data sets from breast and non-small cell lung cancer, suggesting these markers may enrich for tumor-reactive CD4 $+\mathrm{T}$ cells in many cancers.

Conclusions These results identify a subset of tumor infiltrating conventional CD4 $+\mathrm{T}$ cells in melanoma that are enriched for reactivity to tumor antigens and exist in multiple phenotypic states. Correlations of the presence of these cells with the frequency and phenotype of other immune cells suggest roles for these tumor antigen-specific CD4+ T cells in providing CD8 $+\mathrm{T}$ cell help, driving recruitment and maturation of $\mathrm{B}$ cells, and activating macrophages. Isolating such cells based on their unique phenotype and utilizing them for adoptive therapy could alter the tumor microenvironment for therapeutic benefit.

Ethics Approval All Patient samples in this study were obtained from patients who signed informed consent in a study approved by the institutional review board of the Fred Hutchinson Cancer Research Center (protocol \#2643). 